\title{
Review of the Effectiveness of Impulse Testing for the Evaluation of Cable Insulation Quality and Recommendations for Quality
}

\section{Testing}

\author{
Adrian Coughlan \\ Technological University Dublin, adrian.coughlan@mydit.com \\ Joseph Kearney \\ Technological University Dublin, joseph.kearney@tudublin.ie \\ Tom Looby \\ ESB Networks Ireland, Tom.Looby@esb.ie
}

Follow this and additional works at: https://arrow.tudublin.ie/engscheleart

Part of the Computer Engineering Commons, and the Electrical and Computer Engineering Commons

\section{Recommended Citation}

Coughlan, A., Kearney, J., Looby, T. (2018) Review of the Effectiveness of Impulse Testing for the Evaluation of Cable Insulation Quality and Recommendations for Quality Testing ,2018 53rd International Universities Power Engineering Conference (UPEC) 4-7 September,Glasgow, Scotland.

This Conference Paper is brought to you for free and open access by the School of Electrical and Electronic Engineering at ARROW@TU Dublin. It has been accepted for inclusion in Conference papers by an authorized administrator of ARROW@TU Dublin. For more information, please contact arrow.admin@tudublin.ie, aisling.coyne@tudublin.ie, gerard.connolly@tudublin.ie.

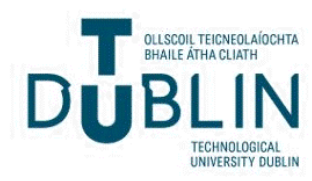




\section{Review of the Effectiveness of Impulse Testing for the Evaluation of Cable Insulation Quality and Recommendations for Quality Testing}

\author{
Adrian Coughlan \\ Dublin Institute of Technology \\ Ireland \\ adrian.coughlan@mydit.com
}

\author{
Dr. Joseph Kearney \\ Dublin Institute of Technology \\ Ireland \\ joseph.kearney@dit.com
}

\author{
Tom Looby \\ ESB Networks \\ Ireland \\ tom.looby@esb.ie
}

\begin{abstract}
This project investigates impulse breakdown testing as a means of determining the as constructed standard of MV power cable. A literature survey is undertaken to elucidate the place of this test in an overall cable test regime and to determine the factors that impact on the performance of the test method. Testing was undertaken on ESB Networks cables to establish if a merit order ranking was feasible based on this test and to determine if the test could detect defects in the inner semiconducting layer. Based on this, conclusions and recommendations are made regarding the overall applicability and usefulness of this test.
\end{abstract}

\section{INTRODUCTION}

The life expectancy and reliability of operation of an XLPE cable depends greatly on the "as manufactured" extrusion standard of the insulation and semi-conductive layers. The probability of cable faults due to insulation breakdown increases as the number of defects in these layers increases. To ensure that MV cables supplied to ESB Networks meets its reliability and lifetime expectations, the cables undergo a number of test stages. These are shown below:

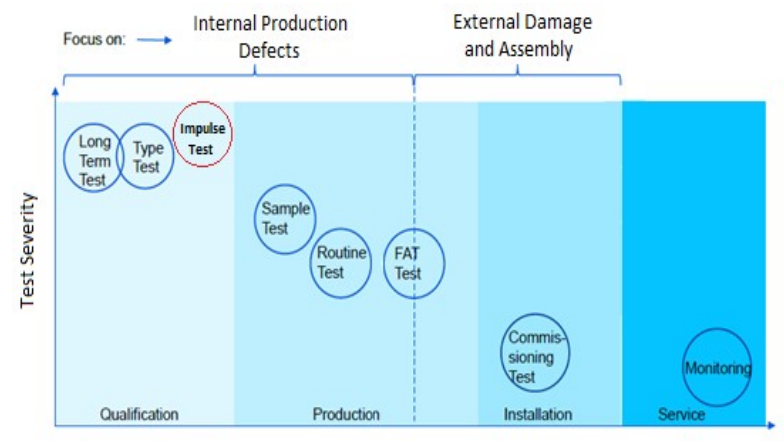

Fig. 1. Test Philosophy

The first test stage is the qualification stage which is carried out on prototype cable samples to confirm that the cable design proposed meets lifetime endurance requirements set out in the cable specification. During this stage samples of proposed cable designs are subject to long term ageing, under thermal cycling and elevated voltage stresses, to ensure that the design of the cable meets the required long-term performance requirements. ESB Networks specifies long term testing for its medium voltage cables using the CENELEC Accelerated Ageing procedure whereby cables are subjected to $3 \mathrm{Uo}$ at $50 \mathrm{~Hz}$ for two years or at $500 \mathrm{~Hz}$ for 3 months.

In addition, Type Test tests are undertaken at the qualification stage by ESB to ensure that the cable design meets detailed IEC and ESB Networks electrical and material requirements Specification requirements. The electrical type tests based on IEC 60840 for $52 \mathrm{kV}$ cables and IEC 60502 for $20 \mathrm{kv}$ cables consist of the following sequence of tests:

- Partial discharge measurement

- Bend test

- Partial discharge test

- Impulse withstand test

- Heat cycle voltage test

- Tan delta measurement

- AC Power frequency test.

The qualification stage is followed by Routine AC and PD tests on production lengths, additionally in the case of $20 \mathrm{kv}$ cables a four-hour AC voltage test on a sample cable length. The commissioning test stage now generally consists of $\mathrm{AC}$ testing, performed at frequencies between $0.1 \mathrm{HZ}$ and $50 \mathrm{~Hz}$ with PD monitoring for long circuits, for relatively short circuit lengths, a soak test is normal.

When cables pass standard Type and Sample and Routine tests outlined by the IEC, the margin by which the cables have passed these tests is unknown to ESB Networks. 
For this reason, impulse breakdown step testing and elevated voltage Type testing was investigated in this project to determine the usefulness of impulse breakdown step testing in relation to;

- Providing a useful merit ranking of different suppliers cables

- Providing insight into the expected performance of cables in long term ageing tests and Type tests

- Indicating the degree of ageing of cables aged in service.

- Detecting manufacturing defects such as gaps in the inner semicon layer

- Indicating a least cost way by which the impulse breakdown performance can be increased

Before discussing each of the above applications of impulse breakdown testing, it is useful to review the factors which affect impulse breakdown strength factors.

\section{LITERATURE REVIEW OF XLPE IMPULSE BREAKDOWN STRENGTH FACTORS}

\section{Influence of Repetitive Impulse Shots}

Fig. 2. below shows the relationship between impulse level and number of impulses.

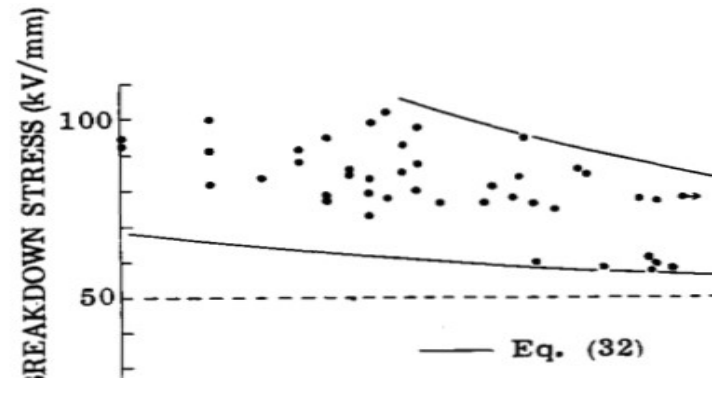

Fig. 2. Minimum Breakdown Voltage

There is a threshold impulse voltage level (approximately $60 \%$ of breakdown voltage). below which the cable insulation will withstand repeated impulses indefinitely. Fig. 2 also shows that there is an aging effect during the test process if the magnitude of the impulse level is close to breakdown level -see the top curve in the diagram.

Apart from this reference, most other references state that there is no ageing effect arising from repeated impulses at breakdown level; however, uncovering specific research which underpins this assumption was not found to be possible.

\section{Influence of Cable Test Temperature}

A temperature of $95^{\circ} \mathrm{C}-100^{\circ} \mathrm{C}$ is specified for impulse testing within the IEC 60540 and 60840 Type test standards. In T.U. Graz impulse breakdown tests, the cable samples are tested at room temperature. This reduces both the costs and complexities of testing. A temperature compensation factor can be used for $20 \mathrm{kV}$ and $52 \mathrm{kV}$ cable to roughly convert results at room temperature to those at $95^{\circ} \mathrm{C}-100^{\circ} \mathrm{C}$. The temperature conversion factor, based on experimental evidence, is 1.25 for negative polarity impulses and $37 \%$ for positive polarity impulses. [5] The 1.25 factor is used extensively in Japanese cable qualification tests and cable insulation thickness design.

\section{Influence of Steepness of Impulse Wave Front}

The rise time, or the steepness of the front of the impulse wave is another factor that impacts on the impulse testing breakdown level. The time for the impulse wave to reach its peak and the impulse wave tail off time are controlled by a pair of resistors. The basic arrangement per Marx impulse generator stage is modelled as follows:

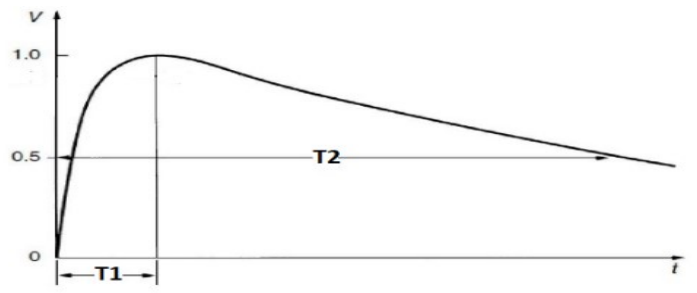

Fig. 3. Impulse Generator Setup and Resultant Waveform [1]

The steepness of the front of the wave (T1) and (T2) are determined by the value of resistors used in the test circuit. As impulse breakdown strength can vary with wave front steepness as well as equipment tolerances and human operator factors, the waveshape should accompany breakdown test results and also be considered when comparing one set of test breakdown results with another.

\section{Effect of Increased Volume on Breakdown Strength}

The probability of defects in insulation increases in line with the increase in cable insulation volume as shown in the graph below. As a result, the impulse breakdown strength decreases significantly as insulation volume increases. Japanese research indicates that average insulation stress per $\mathrm{mm}$ can be used to describe impulse breakdown performance levels.

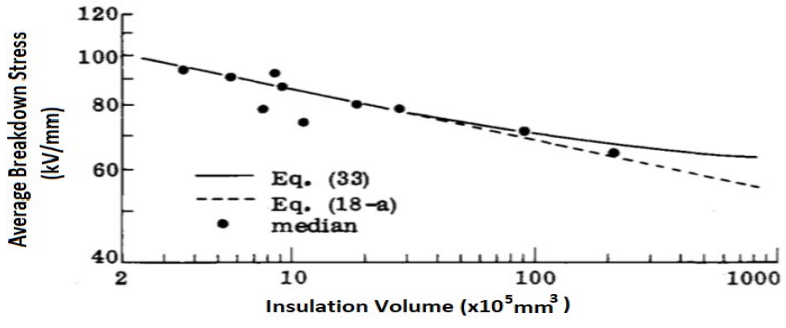

Fig. 4. Relationship of Impulse Breakdown Stress and Volume [1] 


\section{Location of Impulse Breakdowns in the Cable Core}

As can be seen in Fig. 5, the proportion by volume of breakdowns originating in the inner semi con layer (conductor shield) is fewer than in the XLPE insulating layer. The majority of the breakdowns originated at contaminants within the insulation layer.

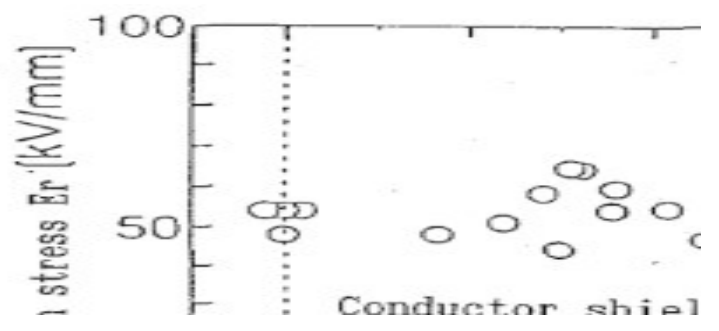

Fig. 5. Cable Failure Position Within Cable Core [3]

From the plot above, the proportion by volume of breakdowns originating in the inner semi con layer (Conductor shield) is fewer than in the XLPE insulating layer. The majority of the breakdowns originated at contaminants within the insulation layer. [4]

\section{Effect of Impulse Polarity on Breakdown Strength}

In accordance with the T.U. Graz specifications for extended impulse testing, all impulses applied to the cable are of negative polarity. The IEC standard for Impulse testing involves application of usage of ten positive impulses and ten negative impulses. The dual polarity approach of IEC is more severe than single polarity testing and a severity factor of $14 \%$ is quoted in the literature to account for this factor. [5]

\section{Effect of Relaxation of XLPE Mechanical Extrusion Stresses on Impulse Withstand Voltage}

After XLPE cable extrusion residual mechanical stress can be produced within the insulation volume as a result of the heated XLPE cooling unevenly after the extrusion process. Online relaxation can relieve this stress by reheating the outer insulation, Based on 14 samples, 7 relaxed and 7 unrelaxed, the Weibull distributed impulse breakdown results showed an increased impulse breakdown strength of $22 \%$ in the stress relaxed cables relative to the unrelaxed cables i.e. the impulse breakdown value increased from $1100 \mathrm{kV}$ to $1346 \mathrm{kV}$ [3].

\section{Background to Step Impulse Breakdown Testing}

A step Impulse breakdown test method and protocol for MV cables was developed by the University of Graz in the early 2000's. This test protocol is used by utilities in Austria, Hungary and Germany as a Specification conformance check. One of the chief advantages of this method, relative to AC endurance step testing, is that it avoids the use of de ionized water terminations. Such terminations are significantly more demanding in terms of equipment and installation resources than the simple and fast cable sheath stripping employed by the TU Graz method.

In the TU Graz method, the voltage grading is controlled via the outer semi-conductive layer which forms a lattice network with the capacitance of the cable. The stress grading is shown in Fig. 8 below; the peak voltage is plotted as a function of the distance from the grounded shield. The voltage stress in the insulation is almost reduced to a linear form.

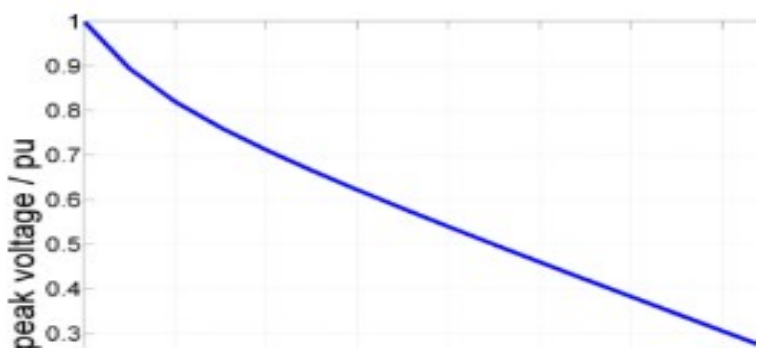

Fig. 6. Voltage Stress in the Area of Voltage Grading

The risk of damaging de ionized water terminations is also eliminated. In addition, the TU Graz test is performed at room temperature, thereby requiring no heating and heating control circuits, which adds to the simplicity of the method.

The other advantage of impulse testing is that the damage at the failure point is limited relative to AC step breakdown as shown by the insulation breakdown in the silicone oil below permitting examination of the failure point and its location.

\section{TEST PROCEDURE}

In the TU Graz testing protocol, five impulses of negative polarity are applied to the cable samples, starting with an impulse magnitude of $350 \mathrm{kV}$. The voltage is then increased in $50 \mathrm{kV}$ steps and five more impulses delivered. This test cycle is then repeated until there has been a breakdown in cable insulation or flashover at the termination ends. When a termination flashover occurs, the end termination flashover resistance can be improved to allow the testing to continue. If these attempts at avoiding flashover are unsuccessful, the test on that sample is terminated. [4] Five samples of ten meters effective test length are tested in each test session.

\section{Cable Preparation}

The step impulse voltage test is carried out on minimum five samples of XLPE insulated cables. The cables tested in this project are all single core aluminium cables with stranded copper 
screen wires and PE over sheath. Each sample was prepared to the following specification.

\section{Test Frequency}

The impulse voltage magnitude and wave front time and the tail off time must be calibrated in the impulse generator by delivering a series of voltage impulses and recording the voltage level and waveform.

\section{TEST PROCEDURE}

The step impulse test is implemented using a suitably rated impulse generator connected via a bare conductor to the test cable length which is suspended from a gantry crane using a polymeric rope and earthed at one or both sides to the test hall earth grid as shown below.

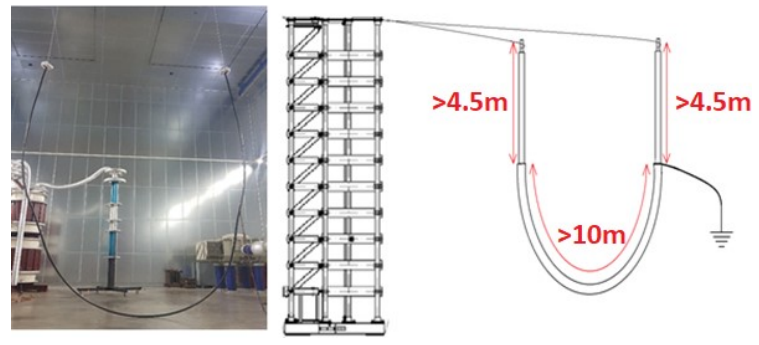

Fig. 7. Test Setup

\section{ESBN Threshold for Passing Impulse Breakdown Tests}

The pass threshold for $20 \mathrm{kV}$ cable is set at $900 \mathrm{kV}$ and $1200 \mathrm{kV}$ for $38 \mathrm{kV}$ cable, using a $50 \mathrm{kV}$ impulse step regime. The $20 \mathrm{kV}$ pass level was based on the average value of TU Graz 63\% Weibull values obtained from a population of 60 cable tests the $52 \mathrm{kV}$ cable impulse breakdown pass level was determined based on ESB Networks results to date. Due to the insulation volume effect, the probability of defects increases with insulation volume. As result the threshold pass voltage for $52 \mathrm{kV}$ cable is less than what the insulation thickness ratio between $52 \mathrm{kV}$ and $20 \mathrm{kV}(10 \mathrm{~mm}: 5.5 \mathrm{~mm})$ i.e. approx. factor of 2 would imply.

\section{Test Frequency}

For ESB Networks cable term contracts which typically have a duration of five years, the impulse to breakdown test samples are taken at six-month intervals from standard production. This ensures even distribution of impulse test samples over the contract period and facilitates trend analysis of the results over time.

The IEC 60502 and 60840 standards require that cable be subjected to 20 impulses (10 positive and 10 negative) at $125 \mathrm{kV}$ for $20 \mathrm{kV}$ and 20 impulses $250 \mathrm{kV}$ for $52 \mathrm{kV}$ at $95-100^{\circ} \mathrm{C}$. By aggregating the 20 impulse steps associated with each of the above impulse breakdown pass levels and adjusting them to compensate for testing at room temperature (1.25) [1] and for testing using negative polarity only (14\%) [2], the above step impulse breakdown pass levels of $900 \mathrm{kV}$ and $1200 \mathrm{kV}$ can be compared with equivalent impulse voltage levels specified in IEC Type tests for $110 \mathrm{kV}$ and $170 \mathrm{kV}$ cables respectively. See severity diagram below. It can therefore be seen that the above pass levels for impulse step test breakdown are onerous and are a stringent test of the cable core as manufactured.

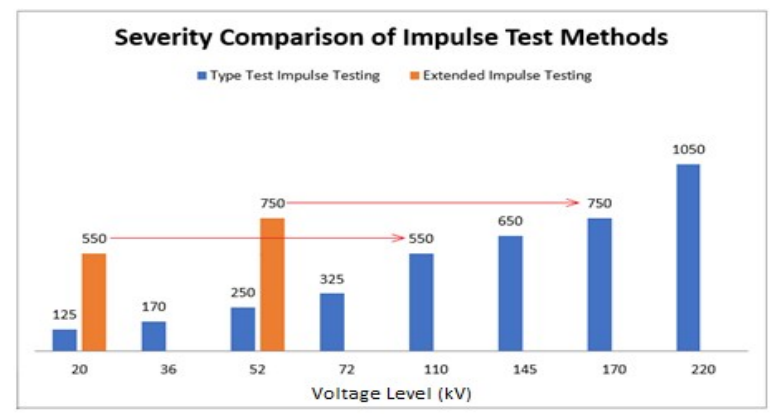

Fig. 8. Severity Comparison

Merit Ordering of Cables

\begin{tabular}{|l|l|l|l|}
\hline \multirow{2}{*}{ Order } & \multicolumn{3}{|c|}{ Cable Breakdown Results (20kV) } \\
\cline { 2 - 4 } & Cable No. & 63\% Value & Pass Mark (900kV) \\
\hline 1 & Cable 6 & $1020 \mathrm{kV}$ & Pass \\
\hline 2 & Cable 7 & $955 \mathrm{kV}$ & Pass \\
\hline 3 & Cable 4 & $942 \mathrm{kV}$ & Pass \\
\hline 4 & Cable 8 & $775 \mathrm{kV}$ & Fail \\
\hline 5 & Cable 5 & $701 \mathrm{kV}$ & Fail \\
\hline
\end{tabular}

Table 1. 20kV Cable Breakdown Results

\begin{tabular}{|l|l|l|l|}
\hline \multirow{2}{*}{ Order } & \multicolumn{3}{|c|}{ Cable Breakdown Results (38kV) } \\
\cline { 2 - 4 } & Cable No. & 63\% Value & Pass Mark (1250kV) \\
\hline 1 & Cable 1 & $1545 \mathrm{kV}$ & Pass \\
\hline 2 & Cable 2 & $1386 \mathrm{kV}$ & Pass \\
\hline 3 & Cable 3 & $1318 \mathrm{kV}$ & Pass \\
\hline 4 & Cable 4 & $1136 \mathrm{kV}$ & Fail \\
\hline
\end{tabular}

Table 2. 38kV Cable Breakdown Results

From Tables 1 and Table 2 above, it can be seen that comparisons can readily be drawn between the cable breakdown voltages for each voltage level. This allows for a simple merit ordering system to be established. Not only does this table show the order by which the cables are ranked, but also shows the reserve on the cable above the pass value. It is expected that a larger reserve should correspond to a longer life expectancy in the cable. Testing on $20 \mathrm{kV}$ cables shows a range from $701 \mathrm{kV}$ to $1020 \mathrm{kV}$, the highest result having a reserve of $120 \mathrm{kV}$ above pass 
level. Similarly, for the $38 \mathrm{kV}$ test set, there is a range from $1136 \mathrm{kV}$ to $1545 \mathrm{~V}$ with the highest having a reserve of $345 \mathrm{kV}$ above the required pass levels.

\section{ANALYSIS OF RESULTS}

Indicator of Performance in Accelerated Ageing and Type Tests

To provide an insight into whether good performance in the Impulse Breakdown Test would translate into good performance in $\mathrm{AC}$ ageing tests, an elevated voltage type test was conducted on a $52 \mathrm{kV}$ cable $(30 \mathrm{kV}$ U0) tested to IEC $110 \mathrm{kV}$ Voltage level.

The list of tests performed per IEC 60840 were:

- Bend Test.

- PD test at $1.5 \mathrm{Uo}(96 \mathrm{kV})$

- Heat Cycle at $2 \mathrm{Uo}\left(128 \mathrm{KV}\right.$ at $\left.95^{\circ} \mathrm{C}\right)$

- Impulse Voltage Test $10+\& 10-\left(550 \mathrm{KV}\right.$ at $\left.95^{\circ} \mathrm{C}\right)$

- PD Test

- AC Power Frequency Test at 2.5 Uo $(128 \mathrm{kV})$

The cable passed the full suite of elevated voltage type tests for the same design of XLPE cable as had passed the Impulse Breakdown step test. This indicates that good performance in the impulse breakdown herald's good performance in long term ageing tests as well as in type tests.

\section{Determining Method of Achieving Higher Breakdown}

\section{Strength Cable Core}

The results for impulse tests can be used to determine the most economical way to improve the impulse breakdown strength. For example, the results can be examined for breakdown location using a silicone oil bath or other investigation method. From that analysis one can determine if it is better overall, in terms of cost and standardization to replace the cable with a higher specification grade of XLPE insulation or alternatively replace the inner semiconducting layer or layers. This is clearly shown by Wald and Woschitz in the paper "Influence on the Impulse Strength of XLPE-insulated Medium Voltage Cables for Different Composites". [4]

Usefulness of Indication of the Degree of Ageing of Cables Aged in Service on the Network

This method was used to evaluate the effect of rejuvenation fluid on samples of $20 \mathrm{kV}$ cable extracted from an Austrian network before and after the treatment. The withstand voltage increased from approximately $300 \mathrm{kV}$ to $475 \mathrm{kV}(+55 \%)$ for one cable circuit and from $412 \mathrm{kV}$ to roughly $512 \mathrm{kV}$. The withstand voltage of the treated cable was very close to the withstand voltage of new $20 \mathrm{kV}$ XLPE insulated cables in the TU Graz impulse breakdown strength database which are in the range of $600-700 \mathrm{kV}$.

In 2010 ESB Networks impulse tested $52 \mathrm{kV}$ cable samples which had experienced 17 years of operation. The breakdown results obtained using the TU Graz procedure averaged $713 \mathrm{kV}$. When this result is processed and translated into equivalent IEC Type test impulse pass values, it exceeds the $250 \mathrm{kV}$ BIL requirement by a significant margin.

Detecting Defects Such as Gaps in the Inner Semicon Layer For the purpose of determining the effectiveness of impulse breakdown testing in detecting the presence of serious cable core defects such as missing sections of inner semicon, ESB Networks arranged to manufacture a $200 \mathrm{~m}$ length of $52 \mathrm{kV}$ cable with stranded $630 \mathrm{AL}$ conductor and 10mm XLPE insulation. The cable was routine and heat cycled tested at $52 \mathrm{kV}$ level (2xUo) and passed all the IEC tests satisfactorily. The test also included a $\mathrm{HV}(4 \mathrm{xUo})$ test for 4 hours which was passed successfully.

However, the cable failed the Impulse breakdown test at 450 $\mathrm{kV}$ which was well below the $1300 \mathrm{kV}$ level expected for this cable type. This clearly shows the effectiveness of the step impulse test in detecting such defects.

\section{CONCLUSIONS}

It was concluded that overall impulse breakdown step testing is a, quick useful and economic testing tool for investigating the "'as built"' standard of MV cable insulation and semiconducting layers, when used in conjunction with other testing and evaluation strategies by both cable engineers and suppliers.

It can be seen from the results outlined in Table 1 and 2 - Merit Order of Results, that impulse testing to breakdown provides a clear distinction between impulse performance of cables supplied to ESB Networks by various suppliers. Taken in conjunction with CENELEC long-term ageing results and, it should therefore prove to be a useful test measure to enable merit ordering of MV cables, provided that due consideration is given to the fact that only a small proportion of all cable production is being tested and to the fact that impulse test results should be carefully compared based on the factors mentioned in the literature review above.

It is also concluded that if a cable performs well on impulse breakdown tests, then this performance will be replicated in $\mathrm{AC}$ long-term ageing tests such as CENELEC two-year tests at 50 $\mathrm{Hz}$ for water treeing. 
It is also concluded that this test can provide useful information when creating a balanced cable design including as to whether to upgrade insulation grade or spend money on upgraded semiconducting layers or both.

Taken on the basis of ESB Networks tests on a $52 \mathrm{kV}$ cable deliberately constructed without an inner semicon on a stranded $630 \mathrm{sq} \mathrm{mm}$ conductor that Impulse breakdown tests were effective in identifying this defect when PD tests and IEC type tests were successfully passed. This success was based on having access to a database of results made up of test results from ESB Networks own test programme and very useful TU Graz published impulse breakdown limit guidelines against which we could compare results with, namely $600 \mathrm{kV}$ cut off for $20 \mathrm{kV}$ cables

Based on the literature survey on impulse testing, it is concluded that the factors that affect insulation breakdown levels in impulse testing to breakdown are as follows;

- Steepness of the front wave

- Average stress on the insulation and volume of insulation

- Temperature of test cable

- Polarity of the impulse waveform

- Mechanical stresses resulting from the extrusion process

- Presence of extrusion by product gases in the insulation

\section{FURTHER RECOMMENDATION}

Standardising the procedure taking into account factors which can impact on the results is considered desirable. These recommendations were broken down into two main categories: standardising test procedures and reducing flashovers. The recommendations are as follows:

\section{Standardising the Wave Front Time}

It is recommended that as far as practically possible, that identical values of rise time are used to enable meaningful comparison of test results.

Published Database of Results and Inclusion of the Impulse Breakdown Test in CENELEC Standards

It is recommended that a database of typical results be published to guide cable utilities in relation to typical breakdown ranges for different cable voltages. It is felt this would be helpful to utilities especially if typical values for aged cables were also included. It is also felt that the TU Graz test could usefully be included in the CENELEC test compendium for MV cables.
The main issue that occurs during impulse breakdown testing is the risk of termination flashovers. The following recommendations are made in relation to avoidance of this issue.

Final Value Focused Testing

This is proposed in an effort to reduce the step range of impulse testing. This could be done by following the standard University of Graz procedure for the first cable i.e. starting at the normal voltage and incrementing at $50 \mathrm{kV}$ intervals until breakdown. Then, when the breakdown level for the first sample is found, the tests for the remaining four cores could start at approximately $20 \%$ lower than the breakdown voltage for the first sample.

\section{Use of Corona Rings}

To use aluminium flexi ducting formed into a donut shape and place around the conductor connected to the impulse generator. This mitigates the effects of sharp corners in the bare impulse generator conductor and in the cable end connector.

Use of Semi-con Tape

Wrap semi-con tape around on areas of bare impulse generator conductor and shield wire. This again reduces the probability of concentrated localised charges flashing over during testing.

\section{Use of Water Tubes}

Use of water tubes to provide a more even charge at the cable screen earth interface. The water tubes are taped to the cable above the grounded shield.

\section{REFERENCES}

[1] K. S. P. N Narayan, "Production of Standard Waves with a $3000 \mathrm{kV}$ Impulse Generator," Journal of the Indian Institute of Science, vol. 34, no. 2, pp. 113-127, 1952.

[2] D. B. M. ,. D. L. K. Andre E. Lux, "The Effect of SteepFront Short-Duration Pulses on Polyethylene Cable Insulation," in Energy and Information Technologies in the Southeast., IEEE, Columbia, SC, USA, 1989.

[3] R. W. a. C. S. D. W. G. Knollseisen, "Influence on the impulse strength of XLPE-insulated medium voltage cables for different composites," in IEEE, Turin, Italy, 2005.

[4] R. M. H. M. \&. S. C. Woschitz, "Quality Control of XLPE Cables by means of Impulse Voltage Test," International Symposium on Electrical Insulating Materials 2005, vol. 2, pp. 526-529, 2005.

[5] P. M. PINET Alain, "A1.1 Dielectric performances of the $20 \mathrm{kV}$ XLPE cable used in France," in JiCable 1989 Aging of Extruded Dielectric Cables, Florida, USA, 1989. 